\title{
Implications of local failure on overall prognosis in aggressive prostate cancer
}

\author{
Layth Mula-Hussain ${ }^{1}$, Soumyajit Roy ${ }^{2}$, Shawn Malone ${ }^{1}$ \\ ${ }^{1}$ Radiation Medicine Program, The Ottawa Hospital Cancer Centre, Ottawa, ON, Canada; ${ }^{2}$ Radiation Oncology Branch, National Cancer Institute, \\ Bethesda, MD, USA \\ Correspondence to: Dr. Shawn Malone, MD, FRCPC. Division of Radiation Oncology, The Ottawa Hospital Cancer Centre, 501 Smyth Road, Box \\ 903, Ottawa, ON, K1H 8L6, Canada. Email: smalone@toh.ca. \\ Provenance and Peer review: This article was commissioned by the editorial office, Translational Andrology and Urology. The article did not undergo \\ external peer review. \\ Comment on: Kishan AU, Chu F, King CR, et al. Local Failure and Survival After Definitive Radiotherapy for Aggressive Prostate Cancer: An \\ Individual Patient-level Meta-analysis of Six Randomized Trials. Eur Urol 2020;77:201-8.
}

Submitted Apr 16, 2020. Accepted for publication May 11, 2020.

doi: $10.21037 /$ tau-2020-04

View this article at: http://dx.doi.org/10.21037/tau-2020-04

High-risk prostate adenocarcinoma (PCa), consisting of patients with either Gleason Score (GS) of 8 to 10, prostatespecific antigen (PSA) more than $20 \mathrm{ng} / \mathrm{mL}$, or clinical tumor-stage T3 or T4, portends a significant risk of relapse and cancer-specific mortality (1). Compared to low-risk $\mathrm{PCa}$, these patients have a more than 3 -fold increased risk of PCa-related death. The combination of long-term androgen deprivation therapy (ADT) and radiotherapy remains a standard management option for these patients (2). Despite combined modality treatment, a significant proportion of men with high-risk PCa, especially those with relatively aggressive features, eventually relapse after first line treatment (3). Stratification of GS into grade groups (GG) has improved the discriminatory ability of the existing risk stratification system in determining the overall prognosis for all risk groups including the highrisk category (4). In a study by Pierorazio et al., 10-year biochemical relapse-free survival was approximately $63 \%$ and $35 \%$ for patients with GG4 and GG5 disease, respectively (4). Another study by Gnanapragasam et al. showed approximately 5- and 17-times higher risk of prostate cancer-specific mortality (PCSM) in patients with GG4 and GG5 PCa, respectively (5). The high relapse rates highlight the importance of further optimization of treatment for men with GG4 and GG5 PCa.

We read with great interest the individual patient-based meta-analysis reported by Kishan et al. (6) in this context. A total of 992 patients were included in the study - 593 with GG4 and 399 with GG5 PCa. Patients in this meta-analysis were originally enrolled and treated with radiotherapy and ADT in one of the following 6 randomized trials: Radiation Therapy \& Oncology Group (RTOG) 8531 (7), RTOG 8610 (8), RTOG 9202 (9), European Organization for Research and Treatment of Cancer (EORTC) 22863 (10), EORTC 22961 (11) and EORTC 22991 (11) (Table 1). Considering local failure as a time-varying covariable, the authors demonstrated an approximate doubling of the risk of distant metastasis and PCSM in patients who had local failure. Using a multi-state disease model and Markov proportional hazard model, they found that transition to local failure was associated with approximately 7 times higher risk of subsequent PCSM and approximately 2 times higher risk of subsequent distant metastasis or death. Long-term ADT in conjunction with radiotherapy yielded reduced risk of PCSM or overall mortality in patients who had experienced local failure. These findings are consistent with a secondary analysis of RTOG 9408 where importance of local control was demonstrated based on a planned biopsy after treatment (12). The presence of a viable tumor on biopsy had a significant association with PSA failure [hazard ratio (HR): 1.7 ; $95 \%$ confidence interval (CI): 1.3-2.1], distant metastasis (HR: 2.4; 95\% CI: 1.3-4.4), cause-specific survival (HR: 3.8 ; 95\% CI: $1.9-7.5$ ), and in those with GS $\geq 7$ even overall mortality (HR: 1.6; 95\% CI: 1.04-2.35), thus lending credibility to the influence of local 
Table 1 Individual trials in the meta-analysis

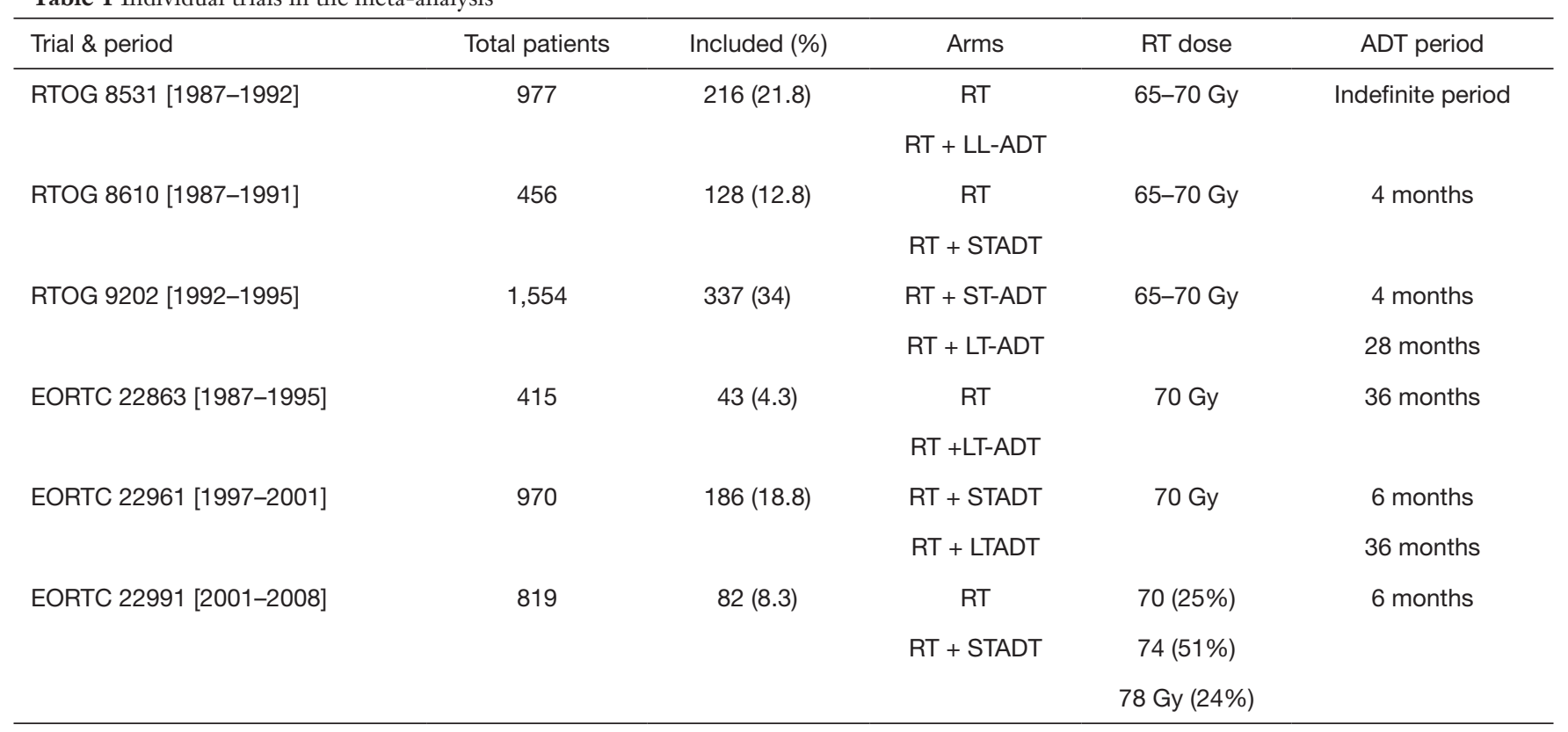

LT-ADT, long-term ADT; ST-ADT, short-term ADT; LL-ADT: lifelong ADT.

control on distant metastasis and PCa-specific survival (12).

The main strengths of this meta-analysis include a large patient population and a unique but robust statistical design. Overall findings allude to an important hypothesis that site of local failure could act as a potential nidus for subsequent distant dissemination in men with GG 4 or 5 PCa. However, the interpretation of the results needs careful attention to some of the underlying limitations of this meta-analysis. These include heterogeneity in the patient population, differences in management protocols, and more importantly variability in the definition of local failure and absence of central pathology review among others. Furthermore, these co-operative group studies were conducted between 1987 to 2008 . As a result, majority of the patients were treated with conventional doses (65-70 Gy) of radiotherapy which might be considered suboptimal compared to the modern standard of dose-escalated external beam radiotherapy (EBRT) (12) or combination of EBRT with brachytherapy boost (13). Similarly, radiotherapy delivery techniques for PCa have evolved over the decade. Use of magnetic resonance imaging (MRI) in radiotherapy treatment planning, delivery of intensity modulated radiotherapy (IMRT) or volumetric modulated arc therapy (VMAT), practice of image-guidance and use of hydrogel spacer between rectum and prostate include some of the notable examples of these advancements $(14,15)$. These technological advancements have resulted in reduced risk of morbidity and have ensured safe dose-escalation. Nonetheless, it remains undefined whether reduction in the risk of treatment-related morbidity potentially improves treatment adherence and thereby oncologic outcome. Extrapolation of the findings of this meta-analysis to the current era should be done with careful consideration to these inconsistencies in radiotherapy dose and treatment techniques.

Some of the additional concerns with this meta-analysis include lack of a priori stratification based on GS in the individual studies, omission of baseline PSA as a covariable from the multivariable regression models or omission of T-stage from the multivariable Markov proportional hazard model for prostate cancer-specific survival (PCSS). Impact of inter-physician and inter-institutional variability in radiotherapy planning and delivery on collective outcomes were not reported and finally, the causes of non-cancerspecific deaths were not mentioned. One should interpret the results in light of the prognostic influence of relative burden of the primary and secondary Gleason patterns on the overall outcome. This has been highlighted in a retrospective study by Huynh et al. They found 2.77-fold increase in the risk of PCSM and 1.75-fold increase in the risk of all-cause mortality for men with GS $3+5 / 5+3$ relative to men with GS $4+4 \mathrm{PCa}(16)$. Similarly, Lim 
et al. found that presence of pathological Gleason $5+4$ adenocarcinoma yielded a significantly inferior biochemical relapse-free survival compared to those with Gleason $4+5$ adenocarcinoma in post-operative biopsy (17). Finally, the results of this study should be construed with due consideration to the use of molecular imaging techniques in $\mathrm{PCa}$ which have shown improved precision in staging of newly diagnosed prostate cancer or identifying relapse after first line treatment (18).

The findings of this meta-analysis lead to some intriguing questions which merit further validation in future randomized controlled studies. One such question is whether escalation of local treatment can spare men with high risk prostate cancer from long-term ADT for 2-3 years which is the backbone of systemic treatment in high-risk prostate cancer $(9,10)$. In ASCENDE-RT study, 12 months of ADT in conjunction with pelvic and low-dose rate brachytherapy boost to prostate yielded commendable biochemical control at 9 years; however, the study does not provide confirmatory evidence on local failure rates (13). In addition, the current study (6) and a previous study from the same group (19) has showed lack of benefit in terms of local control or mortality with continuation of ADT for indefinite period relative to long-term ADT. However, these findings are only hypothesis-generating and need further validation in a randomized clinical trial adequately powered to estimate outcomes such as PCSS or overall survival (OS) with planned stratification for grade group in addition to other relevant prognostic factors.

Intensification of systemic treatment should be revisited considering the findings of this meta-analysis. Findings of the trials from Systemic Therapy in Advancing or Metastatic Prostate Cancer: Evaluation of Drug Efficacy (STAMPEDE) platform favor intensification of systemic treatment in men with locally advanced or high-risk nonmetastatic PCa. In two studies from STAMPEDE platform, where approximately $1 / 3^{\text {rd }}$ of patients harbored locally advanced or high-risk non-metastatic disease, significant OS benefit was obtained with addition of docetaxel and abiraterone to upfront $\operatorname{ADT}(20,21)$. These findings bear important ramifications on the findings of this meta-analysis because patients with GG4 or GG5 PCa could potentially harbor metastases in nodes or distant organs often undetectable by conventional imaging. Further, in RTOG 0521 study (22), where $84 \%$ patients had GS of 8 to 10 , administration of 6 cycles of adjuvant docetaxel after EBRT with concomitant and adjuvant ADT for 2-year improved 6-year OS by approximately 5\% (HR: 0.69; 90\% CI: $0.49-$
0.97). Although addition of chemotherapy improved distant control by $5 \%$ at 6 -year, no information was available on the local failure rates in the two arms. Overall, these findings suggest that intensification of systemic treatment has the potential to synergize with the effect of local treatment which could further improve prognosis in men with highly aggressive PCa. These hypotheses should be tested in future randomized controlled trials.

Along with intensification of systemic treatment, its optimal sequencing with local radiotherapy also merits careful attention. This is even more relevant in the era of hypofractionated radiotherapy for localized PCa where higher dose (3-8 Gy) per fraction is delivered to the prostate and often proximal part of the seminal vesicles (23). Results from two phase III randomized studies have demonstrated that there could be a sequence dependent interaction between $\mathrm{ADT}$ and radiotherapy target volume $(24,25)$. Further studies are necessary to determine the impact of this interaction on outcome in men with GG4 or GG5 PCa and identify those who could potentially benefit from addition of pelvic nodal radiotherapy along with prostate radiotherapy.

To summarize, local control remains an important endpoint in patients with aggressive PCa. Therefore, combination of optimal local and systemic treatment aimed at enhancing local control and thereby cancerspecific or overall survival should be an integral part of for these patients whenever possible. Use of advanced molecular imaging or genomic classifier could aid in better patient selection and maximizing benefit from treatment escalation. Future clinical trials with planned stratification for grade grouping and other relevant prognostic factors will provide further clarity on the ideal treatment regimen for these patients. Such studies could also help unveil potential mechanistic pathways linking local failure with distant metastasis and cancer-specific survival in this patient population.

\section{Acknowledgments}

Funding: None.

\section{Footnote}

Conflicts of Interest: All authors have completed the ICMJE uniform disclosure form (available at http:// dx.doi.org/10.21037/tau-2020-04). SR reports grants from Canadian Association of Radiation Oncology - Uro 
Oncologic Grant, outside the submitted work. The other authors have no conflicts of interest to declare.

Ethical Statement: The authors are accountable for all aspects of the work in ensuring that questions related to the accuracy or integrity of any part of the work are appropriately investigated and resolved.

Open Access Statement: This is an Open Access article distributed in accordance with the Creative Commons Attribution-NonCommercial-NoDerivs 4.0 International License (CC BY-NC-ND 4.0), which permits the noncommercial replication and distribution of the article with the strict proviso that no changes or edits are made and the original work is properly cited (including links to both the formal publication through the relevant DOI and the license). See: https://creativecommons.org/licenses/by-nc-nd/4.0/.

\section{References}

1. Roy S, Morgan SC. Who Dies From Prostate Cancer? An Analysis of the Surveillance, Epidemiology and End Results Database. Clin Oncol (R Coll Radiol) 2019;31:630-6.

2. Anon. Prostate Cancer, Version 2. 2019, NCCN Clinical Practice Guidelines in Oncology in: Journal of the National Comprehensive Cancer Network Volume 17 Issue 5 (2019).

3. Bastian PJ, Boorjian SA, Bossi A, et al. High-risk prostate cancer: from definition to contemporary management. Eur Urol 2012;61:1096-106.

4. Pierorazio PM, Walsh PC, Partin AW, et al. Prognostic Gleason grade grouping: Data based on the modified Gleason scoring system. BJU Int 2013;111:753-60.

5. Gnanapragasam VJ, Lophatananon A, Wright KA, et al. Improving Clinical Risk Stratification at Diagnosis in Primary Prostate Cancer: A Prognostic Modelling Study Beck AH, ed. PLoS Med 2016;13:e1002063.

6. Kishan AU, Chu F, King CR, et al. Local Failure and Survival After Definitive Radiotherapy for Aggressive Prostate Cancer: An Individual Patient-level Meta-analysis of Six Randomized Trials. Eur Urol 2020;77:201-8.

7. Pilepich MV, Winter K, Lawton CA, et al. Androgen suppression adjuvant to definitive radiotherapy in prostate carcinoma--long-term results of phase III RTOG 85-31. Int J Radiat Oncol Biol Phys 2005;61:1285-90.

8. Roach M, Bae K, Speight J, et al. Short-term neoadjuvant androgen deprivation therapy and external-beam radiotherapy for locally advanced prostate cancer: longterm results of RTOG 8610. J Clin Oncol 2008;26:585-91.

9. Hanks GE, Pajak TF, Porter A, et al. Phase III Trial of Long-Term Adjuvant Androgen Deprivation After Neoadjuvant Hormonal Cytoreduction and Radiotherapy in Locally Advanced Carcinoma of the Prostate: The Radiation Therapy Oncology Group Protocol 92-02. J Clin Oncol 2003;21:3972-8.

10. Bolla M, Collette L, Blank L, et al. Long-term results with immediate androgen suppression and external irradiation in patients with locally advanced prostate cancer (an EORTC study): A phase III randomised trial. Lancet 2002;360:103-6.

11. Bolla M, Maingon P, Carrie C, et al. Short Androgen Suppression and Radiation Dose Escalation for Intermediate- and High-Risk Localized Prostate Cancer: Results of EORTC Trial 22991. J Clin Oncol 2016;34:1748-56.

12. Krauss DJ, Hu C, Bahary JP, et al. Importance of local control in early-stage prostate cancer: Outcomes of patients with positive post-radiation therapy biopsy results treated in RTOG 9408. Int J Radiat Oncol Biol Phys 2015;92:863-73.

13. Morris WJ, Tyldesley S, Rodda S, et al. Androgen Suppression Combined with Elective Nodal and Dose Escalated Radiation Therapy (the ASCENDE-RT Trial): An Analysis of Survival Endpoints for a Randomized Trial Comparing a Low-Dose-Rate Brachytherapy Boost to a Dose-Escalated External Beam Boost for High- and Intermediate-risk Prostate Cancer. Int J Radiat Oncol 2017;98:275-85.

14. Bekelman JE, Mitra N, Efstathiou J, et al. Outcomes after intensity-modulated versus conformal radiotherapy in older men with nonmetastatic prostate cancer. Int J Radiat Oncol Biol Phys 2011;81:e325-34.

15. Hamstra DA, Mariados N, Sylvester J, et al. Continued Benefit to Rectal Separation for Prostate Radiation Therapy: Final Results of a Phase III Trial. Int J Radiat Oncol Biol Phys 2017;97:976-85.

16. Huynh MA, Chen $\mathrm{MH}, \mathrm{Wu}$ J, et al. Gleason Score $3+5$ or $5+3$ versus $4+4$ Prostate Cancer: The Risk of Death. Eur Urol 2016;69:976-9.

17. Lim SK, Kim KH, Shin TY, et al. Gleason $5+4$ has worse oncological and pathological outcomes compared with Gleason 4+5: Significance of Gleason 5 pattern. Ann Surg Oncol 2013;20:3127-32.

18. Hofman MS, Lawrentschuk N, Francis RJ, et al. Articles Prostate-specific membrane antigen PET-CT in patients 
with high-risk prostate cancer before curative-intent surgery or radiotherapy ( proPSMA ): a prospective, randomised, multi-centre study. Lancet 2020;395:1208-16.

19. Kishan AU, Wang X, Seiferheld W, et al. Association of Gleason Grade with Androgen Deprivation Therapy Duration and Survival Outcomes: A Systematic Review and Patient-Level Meta-analysis. JAMA Oncol 2019;5:91-6.

20. James ND, Sydes MR, Clarke NW, et al. Addition of docetaxel, zoledronic acid, or both to first-line longterm hormone therapy in prostate cancer (STAMPEDE): Survival results from an adaptive, multiarm, multistage, platform randomised controlled trial. Lancet 2016;387:1163-77.

21. James ND, de Bono JS, Spears MR, et al. Abiraterone for Prostate Cancer Not Previously Treated with Hormone Therapy. N Engl J Med 2017;377:338-51.

Cite this article as: Mula-Hussain L, Roy S, Malone S. Implications of local failure on overall prognosis in aggressive prostate cancer. Transl Androl Urol 2020;9(3):1001-1005. doi:10.21037/tau-2020-04
22. Rosenthal SA, Hu C, Sartor O, et al. Effect of chemotherapy with docetaxel with androgen suppression and radiotherapy for localized high-risk prostate cancer: The randomized phase III NRG Oncology RTOG 0521 Trial. J Clin Oncol 2019;37:1159-68.

23. Roy S, Morgan SC. Hypofractionated Radiotherapy for Localized Prostate Cancer: When and for Whom? Curr Urol Rep 2019;20:53.

24. Roach M, Moughan J, Lawton CAF, et al. Sequence of hormonal therapy and radiotherapy field size in unfavourable, localised prostate cancer (NRG/RTOG 9413): long-term results of a randomised, phase 3 trial. Lancet Oncol 2018;19:1504-15.

25. Malone S, Roy S, Eapen L, et al. Sequencing of AndrogenDeprivation Therapy With External-Beam Radiotherapy in Localized Prostate Cancer: A Phase III Randomized Controlled Trial. J Clin Oncol 2020;38:593-601. 